\title{
A Discussion of the Effect of Musical Context on Perception of Succession of Musical Events, and its Relevance for Philosophical Models of Temporal Experience
}

\author{
MICHELLE PHILLIPS [1] \\ Royal Northern College of Music
}

\begin{abstract}
The article reviewed in this commentary takes philosophical models of temporal experience as its starting point, in an exploration of how an "experience of succession" may be distinguished from a mere "succession of experience". It is proposed that context is the important factor in differentiating the two experiences, rather than duration. Context is accounted for in broad terms, with specific discussion of gesture, performance environment, and mental imagery. The discussion may usefully pave the way for future collaboration between philosophers and psychologists. However, there are multiple fundamental findings in music cognition research, vital to any consideration of the context in which musical experiences occur (e.g. meter, tonality, expectation, familiarity), that could be factored into this discussion. The ideas discussed could be developed with greater consideration of recent empirical studies in music perception. Perhaps then a theoretical model of the experience of succession of musical events could give rise to experimental hypotheses, which may then be tested in order to further refine such models.
\end{abstract}

Submitted 2014 October 16; accepted 2014 November 26.

KEYWORDS: music and time, music cognition, music philosophy

KON (2014, this issue) proposes that theoretical models of time perception may incorporate empirical investigations in order to be useful in future work relating to embodied music cognition. To demonstrate this, the author explores the phenomena of the succession of experience (SoE), and the experience of succession (EoS). Whilst the former describes an awareness of multiple experiences in succession, the latter includes an additional factor - the sensation of one experience as following on from the previous. It is proposed that the two are not distinguished from one another merely by duration (i.e. EoSs as occurring over a short interval, after Phillips, forthcoming), but rather that their distinction depends upon the context of the experience. An example given is that of a $\mathrm{C}$ major broken chord, played either allegro (lasting no more than a second in all), or with an interval of an hour between each of the three notes. It is proposed that the former gives rise to an EoS, as if you were to hear the G4 of this sequence your attention would encompass all of the notes in the chord. It could be argued that this awareness would be direct and not require inference. An SoE, on the other hand, would need inference in the recall of the previous notes, which would appear in consciousness as separate entities. However, once again the extent to which the experience is direct and requires inference is found to be inadequate in accounting for the difference between an EoS and an SoE, particularly when considering the roles of attention and memory. The paper seeks to outline how context may be a more useful tool than duration in distinguishing the two experiences, and how this factor may be included in three philosophical models: 1) the Retentionalist Model captures the notion of the specious present, and proposes that previous events may be "retained" to the extent that they appear simultaneous with the current event, as they have certain degrees of presentness, 2) the Extensionalist Model proposes that experience of events itself is extended in time; there is no sense of an individual instance, 3) the Projectionist Retentionalist Model, in which events are experienced as being connected due to the two events having been experienced together previously. The final section of the article involves the incorporation of Godøy's notion of embodied music cognition into the Projectionist Retentionalist Model. Godøy's research is outlined as proposing that any sound will be accompanied by mental imagery of gesture. Such mental imagery, and the environment in which an event occurs (e.g. a concert hall), may serve as retentions, along with the sound events themselves. The article aims to demonstrate how general philosophical models of time may be informed and developed with reference to findings of empirical studies.

An attempt to support philosophical models with the findings of cognition research is problematic for various reasons, given the history, motivations and purposes of the pursuits in each 
field. The philosophy of time has an extensive history, whereas the study of the perception of time is a relatively recent pursuit. However, this does not render a pursuit to use research evidence to support philosophical models invalid. Indeed, it might be of benefit for philosophers and psychologists to work together. The commentary below will discuss music cognition research which is relevant to the models of time perception presented. In particular, the commentary will detail relevant research and research areas which have not been taken into account in the discussion, before highlighting some of the ways in which empirical research may be further integrated into the philosophical models discussed by the author. The review will begin by considering the perception of musical events in time at the level of the sub- ("timing") and supra-second ("elapsed duration"), and will then outline what "context" may mean in relation to musical events. This second section will focus on research concerning notions of expectation and familiarity in music. These are concepts which could be considered to be implied by the models outlined, but are never explicitly discussed (the notion of expectation features as a relevant factor in the final section of the article, although only in relation to performance space and gesture, rather than with regards to auditory cognition). Although the author states that "we try not to rely on specific theories, hypotheses or accounts at this stage" (p. 215) in the incorporation of some empirical data, it seems that consideration of some of the basic studies and findings of early to recent music cognition research may add a great deal to the ideas explored in this article. The penultimate section will briefly evaluate the relation of Godøy's work on embodied cognition and the incorporation of this into the Projectionist Retentionalist Model, to music cognition research. Finally, this commentary review will conclude with a summary of the perceived usefulness of the models discussed to the field of music cognition.

\section{DURATION}

The point made by the author that duration does not provide an unambiguous way of determining an EoS is important. In seeking to define an upper and lower boundary for the interval between each tone in order for an EoS, the author states that $2 \mathrm{~ms}$ may be an appropriate lower boundary. The reason given is that "the threshold below which we experience two successive auditory tones as simultaneous is 2-3ms" (p. 215). However, more careful consideration of the limits of human hearing may be useful here. Perception of timing between two tones, or "inter onset intervals" (IOIs), is a significant area of exploration in music cognition research. $2 \mathrm{~ms}$ has indeed been demonstrated as the lower boundary for detecting that two sounds are separate, but $20 \mathrm{~ms}$ are required for a listener to extract the order of those sounds, and $50 \mathrm{~ms}$ for a feeling of two separate events (for further summary of the limits of human perception and meter, see London, 2002; London; 2004). Thus, if an EoS requires a sense of one sound following another, then $20 \mathrm{~ms}$ may be a more appropriate lower boundary, and $50 \mathrm{~ms}$ may be the better choice if an EoS requires some sense of individual events.

The proposal of an upper boundary for the interval of time between two sounds, in order for an EoS, is even more problematic. The author proposes that echoic memory (" $2-5$ seconds") is used "as a means of demarcating experiences of different durations" given that "it allows one to retain sensory information after the stimuli has ended" (p. 215). However, echoic memory seems to be one candidate of many for an EoS. Firstly, if it is accepted that a sense of "following on" may be achieved by the retention of the previous sound in working memory, which may vary in length depending on multiple factors, then this upper limit may be much larger. Whether an event is retained in memory does not depend solely on the duration which has elapsed since its occurrence. For example, rehearsal of the information presented may extend its existence in memory. A sound which occurred more than 2-5 seconds previously may still exist in memory (depending on the number and nature of events filling the intervening interval), and hence an EoS may still be experienced when the threshold is larger than echoic memory.

An additional consideration for an upper boundary is that events which may be 2-5 seconds apart from one another are unlikely to be perceived as such, given the nature of psychological time. Such events may be perceived as closer in proximity to one another, or further apart, depending on multiple factors. The perception of time, or elapsed duration, during music listening on the suprasecond level is a relatively recent and growing field of investigation. As musical information unfolds, perception of elapsed duration may vary depending on multiple factors such as the modality (Ziv \& Omer, 2010) or harmonic variation (Firmino, Bueno, \& Bigand, 2009) of the extract or piece of music. Additionally, the extent to which a listener enjoys a piece of music may shape their perception of elapsed duration (Phillips \& Cross, 2010). The assumption that an upper limit should be related to the amount of absolute time between two events neglects to account for the fact that no such record exists in memory of the seconds which have passed. Rather, conditions internal and external to the listener shape this duration. As the author recognises, context must be accounted for. 


\section{CONTEXT}

Context, then, rather than duration, is proposed to distinguish an experience of succession from a succession of experiences. All three of the models discussed (Retentionalist, Extensionalist and Projectionist Retentionalist) attempt to capture the notion that events are only experienced in the context of other neighbouring events. This is no revelation for music cognition. Like visual perception, audition involves the chunking of information into whole forms. Events are grouped together based on principles such as closure, similarity, proximity and continuity. It is this 'grouping' element of cognition which allows recognition of musical phrases, cadences, and themes. Auditory perception also involves the segregation of events into separate individual streams of information - a recording of a concert performance and a cat walking across a piano, if heard simultaneously and at the same listening level, may easily be separated into these individual sound sources (Bregman, 1990). Hence it has long been known that the characteristics of individual auditory events enable them to be identified as belonging to a group with other events nearby.

Music cognition research therefore demonstrates that whether one musical event is experienced as following on from the previous depends to a great extent on context. Length of time between events (which may not necessarily be different tones, but could be rhythms, timbres, dynamic levels etc.) may be one factor which affects experience of succession, but it is indeed not the only one, as the author acknowledges. The remainder of this commentary will examine some specific such factors, focussing on those which are particularly relevant to the philosophical models discussed.

\section{Tonality and Meter}

One limit on the extent to which the proposed development of philosophical models of time perception may be applicable to music cognition is captured in one of the author's own caveats: "we don't discuss F2 [Feature 2 (F2): It has a specific tonal center, i.e., C major] because it is particular to certain 'musical' experiences of auditory succession, rather than experiences of auditory succession generally" (p. 215). The sense of "following on from" in a series of musical events is likely to depend to some extent on the tonality of the sequence. For the last 300 years, tonality has been one of the governing elements in musical composition, albeit with periods beginning in the late $19^{\text {th }}$ century when atonality and other systems were explored and introduced. Prior to this, musical modes dictated the tones which featured together in a sequence, or melody. Tonality is an important context for musical events, and priming experiments have demonstrated that this is a significant dictator of whether one tone is perceived to 'fit' with the previous (Krumhansl, 1990). Moreover, recent research has demonstrated that is it is possible to anticipate the next tone in a musical melody, given the strength of tonal context in anticipation of musical events (Pearce \& Wiggins, 2006).[2] The lack of consideration of tonality limits somewhat the usefulness of the proposed model, as an attempt to account for auditory events which are both musical and non-musical should necessarily factor tonality into the design. The author does recognise this, and proposes that tonality may be one of the contexts which should feature in philosophical models of time.

Many events in music exist not only in a tonal context, but also in a metrical context. The pattern, or rhythm, demonstrated by successive musical events may lead to the inference of meter. This establishment of meter, which facilitates entrainment, is another important consideration in an exploration of how an event, or note, may be deemed to succeed a previous event. Such contexts are relevant to the Retentionalist and Extensionalist Models; degree of 'presentness' of previous events depends on the tonal and metrical relation to the current event, and musical information is not perceived as a series of instances, but rather as grouped chunks of sound.

\section{Expectation and Familiarity}

Both the Retentionalist and Extensionalist Models attempt to account for the notion that experiences generate a sense of expectation of events which may follow others. In music, this expectation may be shaped by tonal and metrical contexts, amongst other factors. Expectation has been known to play a significant role in a response to music since the work of Leonard Meyer in 1956 (Meyer, 1956). Multiple models of musical cognition have built on this, including those of Eugene Narmour (Narmour, 1990) and David Huron (Huron, 2006), all of which attempt to incorporate the sense of expectation which is integral to musical cognition. Theories such as those of tonal pitch space (Lerdahl, 2001) and dynamic attending (Jones \& Boltz, 1989) take the concept of tonal and metrical expectation in musical audition even further. It seems that any attempt to factor music cognition into philosophical models of time must separate musical events from non-musical events, given that musical events exist within frameworks which generate expectation in ways that non-musical events may not.[3] 
Not only is expectation an important consideration in any discussion of how musical events are perceived in time, but the extent to which the sequence of events is familiar to the listener is also relevant. Familiarity may influence sense of elapsed duration during music listening (Bailey \& Areni, 2006), as well as level of enjoyment (Phillips, 2013). The Projectionist Alternative model attempts to account for familiarity. However, given that empirical studies in the field of music cognition have demonstrated convincingly that familiarity is an important context in which musical events are heard, models are needed which develop, rather than reiterate, this notion.

\section{EMBODYING A PHILOSOPHICAL MODEL}

In this final section of the article, theories of music, gesture, and the relevance of performance space and environment are discussed, in relation to the Projectionist Retentionalist Model. The main theories discussed are those of Godøy (2011).

It is not the intention of the current commentary to discuss this section in detail, as the concepts of gesture, auditory imagery and visual information available to the performer and listener have featured in a variety of empirical work in the field of music cognition. It is widely accepted that such notions play in important role in the perception of music. Motion and visual perception play a significant role in any musical experience. They are indeed an important context for musical events. In order for thorough consideration of this aspect of musical experience in any model of temporal perception, there is much additional empirical research which could be consulted. For example, auditory imagery appears to be an important aspect of ensemble performance (Keller \& Appel, 2010), and recent empirical work has suggested that visual cues may be as important, or perhaps even more important, in judgement of music performances (Tsay, 2012).

\section{CONCLUSION}

The author states at the outset that "We believe that these [philosophical] models are in need of systematic empirically-informed refinement" (p. 213). The article aims to incorporate findings from studies of music cognition into philosophical models, including those of Dainton (2010) and Godøy (2011). The author hopes that, by building empirical work into existing models, such models may be useful for future music perception research.

The main discussion in this commentary centres around additional music cognition research which could be incorporated into further discussion of how philosophical models may be supplemented by work in the field of music cognition. Some fundamental empirical studies, such as those exploring the limits of human audition, meter, tonality, expectation and familiarity, are important to any model which attempts to account from how one musical event is deemed to follow on from the previous one. That context is relevant for an experience of succession in music has long been known in music cognition. Indeed, recent research has suggested that the context of the subsequent event may be relevant in music perception, in a theory which has been termed "retrospective perception" (Sears, 2013).

Perhaps the next step in the author's investigation could be to posit a philosophical model based on notions of temporal experience, form a hypothesis as to how its features may be borne out in cognition, and design a series of empirical undertakings to test this model. This may allow for a more thorough and concrete linking of philosophical models of temporal experience, and music cognition.

The article is a starting point for psychologists and philosophers to work together on the perception of musical events in time. Indeed, it is welcomed that music cognition features in such debates concerning the philosophy of time. With its properties of being patterned in time, and comprising of hierarchical tonal and metrical events, music may offer fruitful ground with which to explore further the interrelation of philosophical models and empirical findings relating to temporal experience.

\section{ACKNOWLEDGMENTS}

Many thanks to Jane Ginsborg for her feedback on a draft of this commentary.

\section{NOTES}

[1] Correspondence should be addressed to michelle.phillips@,rncm.ac.uk.

[2] Indeed, software has recently been developed which predicts the probability of each note in a musical sequence, given the musical context which precedes it (Pearce, 2014, https://code.soundsoftware.ac.uk/projects/idyom-project). 
[3] Research has also suggested that an atmosphere containing music may influence perception of time in a different way to an environment with no musical sound (Areni \& Grantham, 2009; Bailey \& Areni, 2006; Noseworthy \& Finlay, 2009), which provides further support for the proposition that a model attempting to account for both musical and non-musical events may be problematic.

\section{REFERENCES}

Areni, C. \& Grantham, N. (2009). (Waiting) Time flies when the tune flows: Music influences affective responses to waiting by changing the subjective experience of passing time. Advances in Consumer Research, 36, 449-455.

Bailey, N., \& Areni, C. S. (2006). When a few minutes sound like a lifetime: Does atmospheric music expand or contract perceived time? Journal of Retailing, 82(3), 189-202.

Bregman, A. S. (1990). Auditory scene analysis. Cambridge, MA: MIT Press.

Dainton, B. (2010). Temporal consciousness. In E.N. Zalta (Ed.), The Stanford Encylopedia of Philosophy. Retrieved from http://ruccs.rutgers.edu/faculty/pylyshyn/Consciousness_2014/StanfordEncyclopedia/consciousnesstemporal_sc.pdf.

Firmino, É. A., Bueno, J. L. O., \& Bigand, E. (2009). Travelling through pitch space speeds up musical time. Music Perception, 26(3), 205-209.

Huron, D. B. (2006). Sweet anticipation: Music and the psychology of expectation. Cambridge, Mass.: London, MIT Press.

Godøy, R.I. (2011). Coarticulated gestural-sonorous objects in music. In A. Gritten \& E. King (Eds.), New perspectives on music and gesture (pp. 67-82). Surrey: Ashgate.

Jones, M. R. \& Boltz, M. (1989) Dynamic attending and responses to time, Psychological Review, 96(3), 459-491.

Keller, P. E., \& Appel, M. (2010). Individual differences, auditory imagery, and the coordination of body movements and sounds in musical ensembles. Music Perception, 28(1), 27-46.

Krumhans1, C. L. 1990. Cognitive foundations of musical pitch. Oxford: Oxford University Press.

Lerdahl, F. (2001). Tonal pitch space, Oxford: Oxford University Press.

London, J. (2002). Cognitive constraints on metric systems: Some observations and hypotheses. Music Perception, 19(4), 529-550.

London, J. (2004). Hearing in time: Psychological aspects of musical meter. Oxford: Oxford University Press.

Meyer, L. B. (1956). Emotion and meaning in music. Chicago: Chicago University Press.

Narmour, E. (1990). The analysis and cognition of basic melodic structures. Chicago: University of Chicago Press.

Noseworthy, T. J. \& Finlay, K. (2009). A comparison of ambient casino sound and music: Effects on dissociation and on perceptions of elapsed time while playing slot machines. Journal of Gambling Studies, 25(3), 331-342.

Pearce, M. T. (2014). Idyom Project. (Website). https://code.soundsoftware.ac.uk/projects/idyomproject. Accessed on 7 th October 2014.

Pearce, M. T., \& Wiggins, G. A. (2006). Expectation in melody: The influence of context and learning, Music Perception, 23(5), 377-405. 
Phillips, I. (forthcoming). The temporal structure of experience. In D. Lloyd \& V. Arstila (Eds.), Subjective time: The philosophy, psychology and neuroscience of temporality. Cambridge: MIT Press.

Phillips, M., \& Cross, I. (2011). About musical time - effect of age, enjoyment, and practical musical experience on retrospective estimate of elapsed duration during music listening. In A. Vatakis et al. (Eds.), Time and Time Perception 2010, LNAI 6789 (pp. 125-136).

Phillips, M. (2013). The role of factors associated with emotion in experience of elapsed duration during music listening. Paper presented at the Society for Music Perception and Cognition (SMPC), Toronto, ON, August 8-11.

Sears, D. (2013). Hierarchies of cadential closure: Effects of training and context. Paper presented at the Society for Music Perception and Cognition (SMPC), Toronto, ON, August 8-11.

Tsay, C-J. (2012). The impact of visual cues on the judgment and perceptions of music performance. Paper presented at the joint International Conference on Music Perception and Cognition and European Sciences for the Cognition of Music (ICMPC-ESCOM), Thessaloniki, Greece, July 23-28.

Ziv, N., \& Omer, E. (2010). Music and time: The effect of experimental paradigm, musical structure and subjective evaluations on time estimation. Psychology of Music, 39(2), 182-195. 